\title{
A Continuous-Discontinuous Approach to Simulate Heat Transfer in Fractured Media
}

\section{Journal Article}

Author(s):

Moonen, P.; Sluys, L.J.; Carmeliet, J.

Publication date:

2011-09

Permanent link:

https://doi.org/10.3929/ethz-b-000160074

Rights / license:

In Copyright - Non-Commercial Use Permitted

Originally published in:

Transport in Porous Media 89(3), https://doi.org/10.1007/s11242-011-9777-y 


\title{
A Continuous-Discontinuous Approach to Simulate Heat Transfer in Fractured Media
}

\author{
P. Moonen • L. J. Sluys • J. Carmeliet
}

Received: 14 June 2010 / Accepted: 30 April 2011 / Published online: 13 May 2011

(C) Springer Science+Business Media B.V. 2011

\begin{abstract}
A macroscopic framework to model heat transfer in materials and composites, subjected to physical degradation, is proposed. The framework employs the partition of unity concept and captures the change from conduction-dominated transfer in the initial continuum state to convection and radiation-dominated transfer in the damaged state. The underlying model can be directly linked to a mechanical cohesive zone model, governing the initiation and subsequent growth and coalescence of micro-cracks. The methodology proved to be applicable for quasi-static, periodic, and transient problems.
\end{abstract}

Keywords Failure - Damage - Cohesive zone model · Partition of unity (PU) . Heat transfer · Continuous-discontinuous framework

\section{List of Symbols}

\section{Latin Symbols}

A Area $\left(\mathrm{m}^{2}\right)$

$A_{\mathrm{i}} \quad$ Amplitude of the periodic temperature fluctuation on the inner wall surface $(\mathrm{K})$

B Matrix containing the derivatives of the finite element shape functions $\left(\mathrm{m}^{-1}\right)$

c Specific heat capacity $(\mathrm{J} /(\mathrm{kg} \mathrm{K}))$

$C_{\mathrm{b}} \quad$ Black body constant $\left(\mathrm{W} /\left(\mathrm{m}^{2} \mathrm{~K}^{4}\right)\right)$

P. Moonen

Katholieke Universiteit Leuven (KUL), Leuven, Belgium

P. Moonen · L. J. Sluys

Delft University of Technology (TUD), Delft, The Netherlands

P. Moonen $(\varangle) \cdot$ J. Carmeliet

Swiss Federal Institute of Technology Zürich (ETH), Zürich, Switzerland

e-mail: moonen@arch.ethz.ch

P. Moonen · J. Carmeliet

Swiss Federal Laboratories for Materials Science and Technology (EMPA), Dübendorf, Switzerland 


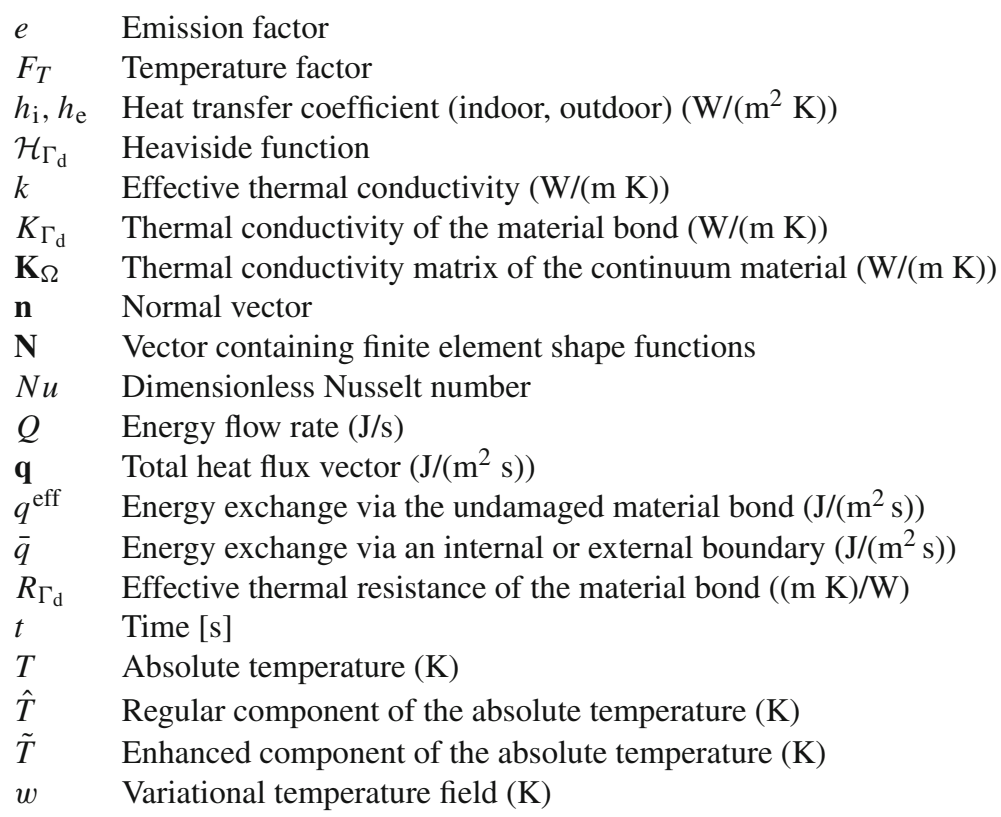

\section{Greek Symbols}

$\alpha \quad$ Relative interface position

$\gamma \quad 1$ Unit meter $(\mathrm{m})$

$\lambda$ Thermal conductivity of the medium inside the discontinuity $(\mathrm{W} /(\mathrm{m} \mathrm{K}))$

$\phi_{\mathrm{i}} \quad$ Phase angle of the periodic temperature fluctuation on the inner wall surface $\left(^{\circ}\right)$

$\rho$ Average mass density $\left(\mathrm{kg} / \mathrm{m}^{3}\right)$

$\omega$ Damage variable

\section{Subscripts}

$\begin{array}{ll}\Gamma & \text { Boundary } \\ \Gamma_{\mathrm{d}} & \text { Discontinuity } \\ \Omega & \text { Body }\end{array}$

\section{Introduction}

Coupled hygro-thermo-mechanical processes are the main cause of physical degradation of porous (building) materials, they are a determining factor in many biological and chemical processes, and they are prevalent in various geophysical applications, like underground storage of nuclear or hazardous waste and geothermal energy extraction. Thermo-mechanical behavior is of importance in the design of micro-electronic devices, especially regarding the connections between materials with different thermal properties. A proper understanding of thermal transport processes in porous media with inclusions, such as fractures or material interfaces, is therefore indispensible. 
Studies during the nineteenth and twentieth century led to the development of a general theory describing the behavior of multi-phase porous continua, based on the principles of mechanics and thermodynamics (De Boer 2000). Although many materials can initially be considered as porous continua, they may degrade over time. Since it is well known that transport processes in porous media can be affected to a large extent by the presence of fractures, a reliable analysis of the risk associated with certain geophysical and civil engineering applications or a prediction of the degradation rate of materials or material interfaces, needs to account for the presence and the potential evolution of the fracture network in the porous medium (Roels et al. 2003). Nowadays, efforts are being made to incorporate effects of crack initiation and propagation into the continuum theory. A trend toward coupled continuousdiscontinuous modeling techniques can be observed.

Regarding the modeling of failure, this idea has been pursued by many authors. Ren and Bićanić (1997) employ an element removal technique to obtain discrete cracks at the final stages of failure. Jirásek and Zimmermann (2001) propose a method in which smeared cracks are combined with embedded discontinuities. Simone et al. (2003) introduce a traction-free discontinuity at the final stages of failure. Similar to Ren and Bićanić (1997), the continuum model governs the softening behavior. Wells and Sluys (2001) make use of the cohesive zone assumption (Dugdale 1960; Barenblatt 1962) and the fracture energy concept (Hillerborg et al. 1976) to describe the gradual formation of cracks in quasi-brittle materials. A traction-separation model governs the non-linear behavior in the fracture process zone and the continuum was assumed to remain elastic at all times. All mentioned approaches have in common that a single moment exists at which the continuous model is replaced by a discontinuous model. The distinct feature of the model proposed by Moonen et al. (2008) is that this transition takes place gradually. This avoids the introduction of a high dummy stiffness or an artificial damage parameter for the non-active cracks, and enhances the robustness of the algorithm.

Parallel to the research on the modeling of failure, mass transfer in fractured porous media has also been the subject of study. At present, the most widespread models used in practice are the equivalent porous continuum model (Wu et al. 1999) and the dual permeability model (Pruess et al. 1999). However, these only describe the geometry of the fracture network in an approximate way and are, therefore, not capable to capture all physics of the flow inside the fracture. This observation stimulated the development of so-called discrete fracture flow models, where the geometry of the fracture network is explicitly modeled (Vandersteen et al. 2003; Carmeliet et al. 2004). The discrete model can be coupled to the continuum transport model by employing interface elements (Segura and Carol 2008), by introducing a mass source term along the length of a discrete fracture (Therrien and Sudicky 1996; Roels et al. 2006) or by inserting a strong (de Borst et al. 2004; Alfaiate et al. 2010) or a weak (Réthoré et al. 2007, 2008) discontinuity in the pressure field.

The literature on heat flow in fractured porous media is rather limited. Often fractures are considered in an approximate way, or adiabatic behavior is assumed, i.e., no energy is exchanged with the surrounding porous material. Heat exchange between the fracture and the surrounding matrix is considered in the work of Secchi et al. (2004). They employ a remeshing strategy to capture the change in topology upon fracture growth. The effect of the fracture on the temperature distribution was shown to be in good agreement with experimental data. Fagerström and Larsson (2008) insert a strong discontinuity in the temperature field to capture the impact of varying topology on the temperature distribution, hereby avoiding the need for remeshing. Unfortunately, the full potential of the proposed method is not shown, since their examples are limited to the case in which the temperature field exhibits a weak discontinuity. 
The aim of this article is to propose a macroscopic model for heat transfer in fractured materials or composites. The model incorporates the gradual change from conductiondominated transfer in the continuum state, prior to crack formation, to convection and radiation-dominated transfer in the damaged state afterward. As shown in Moonen et al. 2010, the proposed model can be directly linked to a mechanical cohesive zone model governing the initiation and subsequent growth and coalescence of micro-cracks. It can also be combined with discrete fracture flow models, describing the transport of fluid-and enthalpy-inside the crack (e.g., Vandersteen et al. 2003; Carmeliet et al. 2004). As such, the proposed theory contributes to the development of more complete models for applications in geophysics, civil engineering, building physics, and micro-electronics.

The article is structured as follows. In the next section (Sect. 2), heat transfer in a fractured porous medium is discussed. First, we introduce the cohesive zone concept. Afterward the use of a discontinuous temperature field is motivated, followed by the derivation of the damage-based discrete constitutive equation for heat transfer. Finally, simplified models for heat transfer across micro-cracks and cavities are given. In Sect. 3, the implementation of the model in a strong discontinuity framework is treated. Both strong and weak form and aspects of discretization and linearization are discussed. The last section (Sect. 4) contains a number of academic examples to illustrate the potential of the proposed modeling strategy.

\section{Heat Transfer in Fractured Porous Media}

\subsection{Cohesive Zone Concept}

In quasi-brittle materials, a transition region exists between the fracture and the undamaged material. In this region, micro-cracks and defects are present, but have not yet coalesced into a discrete fracture. The partially damaged material, therefore, still possesses some degree of cohesion, but generally less than the undamaged material. This transition region is termed the cohesive zone. Cohesive zones exist both in material and in interface failure. The latter is illustrated in Fig. 1a.

(a)

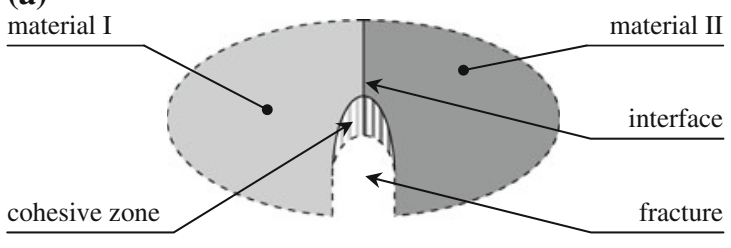

(b)

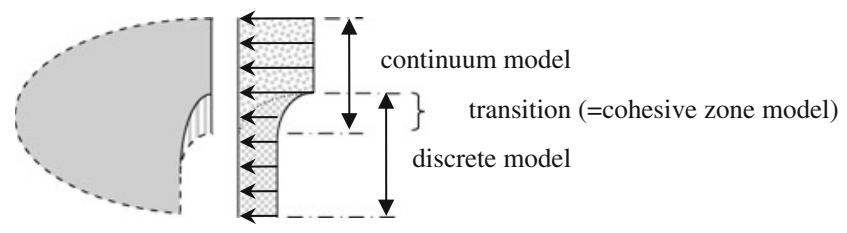

Fig. 1 a Schematic representation of a body $\Omega$ consisting of two materials with indication of the cohesive zone and the fracture domain and $\mathbf{b}$ applied modeling techniques in the different regions 
Successful modeling of the behavior of damaged porous media crucially depends on the adopted modeling technique for the cohesive zone. The approach described in this article starts from the physical observation that the cohesive zone is a transition region between the continuum material on the one hand and the fracture on the other hand (Fig. 1b).

In the continuum material, heat transfer is often modeled as effective conduction. The word effective indicates that the macroscopically observed conductive heat flow results from a combination of conductive flow through the porous material matrix, conductive and convective flow in the fluid occupying the pore space and energy exchange by means of radiation between the pore walls. If an external pressure gradient is exerted on the fluid inside the pore space, the resulting flow provides an additional means to transport energy. At the macroscale, the material can be regarded as a porous continuum, and, since the basic principles of continuum mechanics like continuity and smoothness are fulfilled at this scale, continuum models based on effective conductivity are an adequate modeling technique for this region.

The fracture network can be modeled as a system of interconnected channels through which heat (and mass) transfer can take place. Conduction, convection, and radiation are again the main heat transfer mechanisms. However, because the characteristic width of the flow channels is typically much larger than the characteristic pore size of the surrounding material, heat transfer in the fracture network cannot be modeled as effective conduction. Instead, an explicit description of the individual heat transfer mechanisms needs to be given, based on the actual dimensions and connectivity of the fracture network. Discrete fracture flow models provide such description and are therefore an adequate modeling technique for this region.

In the cohesive zone, we simultaneously encounter continuum and discrete behavior. Close to the boundary with the material domain, effective conduction adequately describes heat transfer, while close to the boundary with the fracture domain, the dominant transfer mechanisms of the fracture domain prevail. The proposed cohesive zone model insures a gradual transition from a continuum model into a discrete model, as the degree of degradation increases (Fig. 1b), and will be presented in Sect. 2.3. The modeling technique for the cohesive zone is formulated irrespective of the choice for a specific continuum or discrete model, and does not introduce artificial parameters or parameters with a debatable physical significance. As will be shown, most existing continuum and discrete models can be incorporated into the generic framework in an efficient and robust way. This allows selecting the best available models for the application under study.

\subsection{A Discontinuity in the Temperature Field}

The temperature distribution $T$ inside an intact porous medium is assumed to be continuous. If a fracture occurs, the overall temperature field remains continuous, although the temperatures at both crack surfaces can be different (Fig. 2a). In that case, the temperature field exhibits a finite gradient over the width of the fracture, i.e., across the fracture domain (Fig. 2b). The temperature distribution in the material domain $\Omega$ is piecewise continuous (Fig. 2c). Mathematically, the latter can be expressed as:

$$
T=\hat{T}+H_{\Gamma_{\mathrm{d}}} \tilde{T} \text { on } \Omega
$$

where $\hat{T}$ and $\tilde{T}$ are smooth, continuous functions on $\Omega$ and $H_{\Gamma_{\mathrm{d}}}$ is the Heaviside step function, used as enhanced approximation basis. The Heaviside function is equal to one for all points $x \in \Omega^{+}$and zero for all other points $x \in \Omega^{-}$, where $\Omega=\Omega^{-} \cup \Omega^{+}$. Equation 1 is based on the Partition of Unity principle. This concept was first formulated by Melenk and Babuška (1996) using a continuous enrichment function. Later, Moës et al. (1999) applied this 


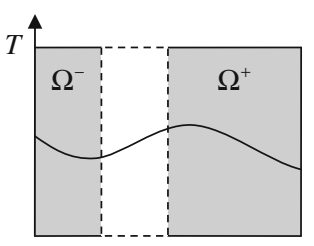

(a) fractured porous medium

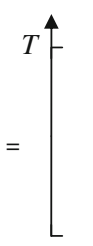

(b) fracture domain

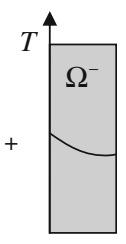

(c) material domain $\Omega=\Omega^{-} \cup \Omega^{+}$

Fig. 2 Temperature distribution inside a the fractured porous medium, and inside the two sub-domains, namely $\mathbf{b}$ the fracture domain and $\mathbf{c}$ the material domain $\Omega=\Omega^{-} \cup \Omega^{+}$

concept to study crack propagation processes, hereby employing a discontinuous Heaviside enrichment function.

\subsection{Damage-Based Discrete Constitutive Equation for Heat Transfer}

\subsubsection{General Derivation}

Consider a partially fractured material interface, as illustrated in Fig. 1. We observe several regions: the two material domains, the fracture and the cohesive zone in front of the crack tip. The cohesive zone contains imperfections, like micro-cracks and voids, which can potentially coalesce and form a macro-crack. This microscopic damage at the interface is schematically depicted in Fig. 3a. The amount of interface damage can be described by means of a scalar damage variable $\omega$, defined as the ratio of the fractured area to the total area of an infinitesimal part $d A$ of the interface. The damage variable $\omega$, therefore, ranges from zero to one, where the case $\omega=0$ corresponds to a locally intact interface, and $\omega=1$ to a complete separation of the two materials along that part of the interface. The magnitude of the damage variable $\omega$ can be determined by means of a (damage-based) mechanical cohesive zone model, like the one proposed in Moonen et al. (2008).

The derivation for the discrete cohesive zone model for heat transfer starts from energy conservation, written for three different positions, namely $\Gamma_{\mathrm{d}}^{-}, \Gamma_{\mathrm{d}}^{+}$, and $\Gamma_{\mathrm{d}}$, which are the interfaces between $\Omega^{-}$and the cohesive zone, $\Omega^{+}$and the cohesive zone, and between the two materials, respectively (Fig. 3a). For an infinitesimal part of $\Gamma_{\mathrm{d}}^{-}$with area $d A$ and outward normal $\mathbf{n}^{-}$, energy conservation reads (Fig. 3b):

$$
\sum Q=0 \rightarrow \mathbf{q}^{-} \cdot \mathbf{n}^{-} d A+q_{\Gamma_{\mathrm{d}}^{-}}^{\mathrm{eff}}(1-\omega) d A+\bar{q}_{\Gamma_{\mathrm{d}}^{-}} \omega d A=0
$$

where $Q$ is the energy flow rate through the surface $d A, \mathbf{q}^{-}$is the heat flux in $\Omega^{-}$at the interface $\Gamma_{\mathrm{d}}^{-}, q_{\Gamma_{\mathrm{d}}^{-}}^{\text {eff }}$ is the amount of energy exchanged per unit area and time between $\Gamma_{\mathrm{d}}$ and $\Gamma_{\mathrm{d}}^{-}$via the undamaged part of the cohesive zone $((1-\omega) d A)$, i.e., the intact material bond, and $\bar{q}_{\Gamma_{\mathrm{d}}^{-}}$is the amount of energy exchanged per unit area and time between $\Gamma_{\mathrm{d}}$ and $\Gamma_{\mathrm{d}}^{-}$, via the damaged part of the cohesive zone $(\omega d A)$, i.e., the microscopic fracture. The latter flux can, e.g., represent the enthalpy flux corresponding to evaporation at the fracture surface. More details on the exchange term $\bar{q}_{\Gamma_{\mathrm{d}}^{-}}$are given in Sect. 2.4. By convention, $q_{\Gamma_{\mathrm{d}}^{-}}^{\text {eff }}$ and $\bar{q}_{\Gamma_{\mathrm{d}}^{-}}$ are positive if energy is added to the interface $\Gamma_{d}^{-}$. The components of $\mathbf{n}^{-}$and $\mathbf{q}^{-}$are positive if they are aligned with the axes of the global coordinate system. 


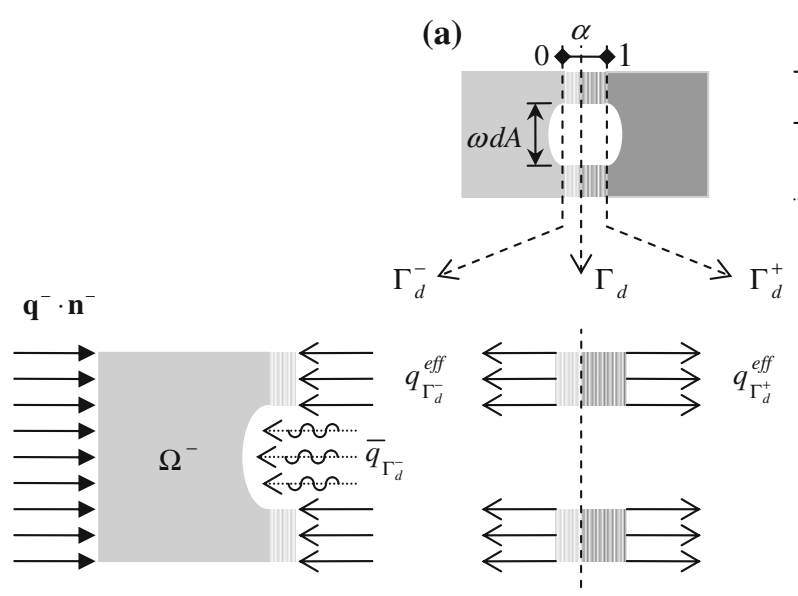

(b)

(c)
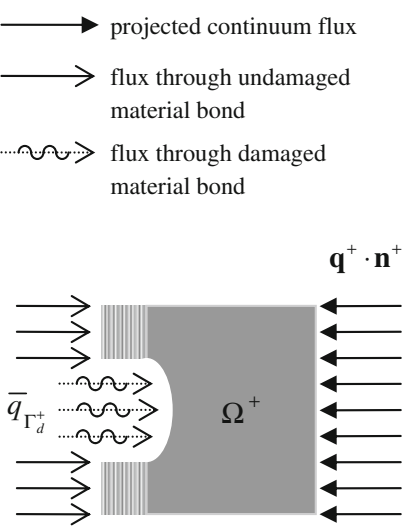

(d)

Fig. 3 a Schematic representation of the damaged material interface. Heat flux equilibrium at the positions $\mathbf{b} \Gamma_{\mathrm{d}}^{-}, \mathbf{c} \Gamma_{\mathrm{d}}$, and $\mathbf{d} \Gamma_{\mathrm{d}}^{+}$

We decompose the effective heat flux $q_{\Gamma_{\mathrm{d}}^{-}}^{\text {eff }}$ into two components, namely (i) a component proportional to the temperature gradient, in magnitude equal to the continuum flux in $\Omega^{-}$at $\Gamma_{\mathrm{d}}^{-}$and (ii) a discrete component, proportional to the difference in temperature between $\Gamma_{\mathrm{d}}^{-}$ and $\Gamma_{\mathrm{d}}$. At this stage, the latter component can be seen as a correction term, to compensate for the unbalance between $q_{\Gamma_{\mathrm{d}}^{-}}^{\text {eff }}$ and the continuum term. Later, it will become clear that this discrete term is damage-related, as is the development of a temperature jump. The term $q_{\Gamma_{\mathrm{d}}^{-}}^{\text {eff }}$ is given by:

$$
q_{\Gamma_{\mathrm{d}}^{-}}^{\text {eff }}=-\mathbf{q}^{-} \cdot \mathbf{n}^{-}+\gamma^{-1} \alpha^{-1} K_{\Gamma_{\mathrm{d}}^{-}}\left(T_{\Gamma_{\mathrm{d}}}-T_{\Gamma_{\mathrm{d}}^{-}}\right)
$$

where the minus sign in front of the heat flux term results from the convention that sink terms are negative. The quantity $\gamma$ follows from dimensional analysis and equals one unit meter. The variable $\alpha$, ranging between zero and one, indicates the relative position of the material interface within the cohesive zone (Fig. 3a). In case $\alpha$ is zero, $\Gamma_{\mathrm{d}}$ coincides with $\Gamma_{\mathrm{d}}^{-}$, in case $\alpha$ is one, the material interface coincides with $\Gamma_{\mathrm{d}}^{+}$. The default value is $\alpha=0.5$. Physically $\alpha$ defines the relative contribution of each of the materials sharing the material interface to the material behavior within the cohesive zone. $T_{\Gamma_{\mathrm{d}}}$ and $T_{\Gamma_{\mathrm{d}}^{-}}$are the temperatures at $\Gamma_{\mathrm{d}}$ and $\Gamma_{\mathrm{d}}^{-}$, respectively. $K_{\Gamma_{\mathrm{d}}^{-}}$is the discrete conductivity of the material bond. $K_{\Gamma_{\mathrm{d}}^{-}}$can be modeled as $\mathbf{n}^{-} \cdot \mathbf{K}_{\Omega^{-}} \mathbf{n}^{-}$, i.e., the projection of the continuum conductivity $\mathbf{K}_{\Omega^{-}}$onto the interface $\Gamma_{\mathrm{d}}^{-}$. Alternatively, an expression for $K_{\Gamma_{\mathrm{d}}^{-}}$can be postulated and determined by means of experiments, or $K_{\Gamma_{\mathrm{d}}^{-}}$can be obtained from upscaling a lower scale simulation in which the degradation of the cohesive zone is modeled in detail.

Substitution of (3) into (2) and rearranging leads to:

$$
\mathbf{q}^{-} \cdot \mathbf{n}^{-}=(1-\omega)\left(\mathbf{q}^{-} \cdot \mathbf{n}^{-}+\gamma^{-1} \alpha^{-1} K_{\Gamma_{\mathrm{d}}^{-}}\left(T_{\Gamma_{\mathrm{d}}^{-}}-T_{\Gamma_{\mathrm{d}}}\right)\right)-\omega \bar{q}_{\Gamma_{\mathrm{d}}^{-}}
$$

Analogously, the equilibrium at $\Gamma_{\mathrm{d}}^{+}$yields (Fig. 3d):

$$
\mathbf{q}^{+} \cdot \mathbf{n}^{+}=(1-\omega)\left(\mathbf{q}^{+} \cdot \mathbf{n}^{+}+\gamma^{-1}(1-\alpha)^{-1} K_{\Gamma_{\mathrm{d}}^{+}}\left(T_{\Gamma_{\mathrm{d}}^{+}}-T_{\Gamma_{\mathrm{d}}}\right)\right)-\omega \bar{q}_{\Gamma_{\mathrm{d}}^{+}}
$$


Energy conservation for the intact part of the material interface $\Gamma_{\mathrm{d}}$, can be expressed as (Fig. 3c):

$$
q_{\Gamma_{\mathrm{d}}^{-}}^{\mathrm{eff}}+q_{\Gamma_{\mathrm{d}}^{+}}^{\mathrm{eff}}=0
$$

Equation 6 allows determining the interface temperature $T_{\Gamma_{\mathrm{d}}}$. To that extent we first insert the expression for $q_{\Gamma_{\mathrm{d}}^{-}}^{\text {eff }}$ (Eq. 3) and an equivalent expression for $q_{\Gamma_{\mathrm{d}}^{+}}^{\text {eff }}$ into Eq. 6:

$$
\left(\mathbf{q}^{-} \cdot \mathbf{n}^{-}+\gamma^{-1} \alpha^{-1} K_{\Gamma_{\mathrm{d}}^{-}}\left(T_{\Gamma_{\mathrm{d}}^{-}}-T_{\Gamma_{\mathrm{d}}}\right)\right)+\left(\mathbf{q}^{+} \cdot \mathbf{n}^{+}+\gamma^{-1}(1-\alpha)^{-1} K_{\Gamma_{\mathrm{d}}^{+}}\left(T_{\Gamma_{\mathrm{d}}^{+}}-T_{\Gamma_{\mathrm{d}}}\right)\right)=0
$$

Now, $\mathbf{q}^{-} \cdot \mathbf{n}^{-}$can be eliminated by means of Eq. 4 and $\mathbf{q}^{+} \cdot \mathbf{n}^{+}$by means of Eq. 5 , leading to:

$$
T_{\Gamma_{\mathrm{d}}}=\frac{\gamma^{-1}\left(\alpha^{-1} K_{\Gamma_{\mathrm{d}}^{-}} T_{\Gamma_{\mathrm{d}}^{-}}+(1-\alpha)^{-1} K_{\Gamma_{\mathrm{d}}^{+}} T_{\Gamma_{\mathrm{d}}^{+}}\right)-\omega\left(\bar{q}_{\Gamma_{\mathrm{d}}^{-}}+\bar{q}_{\Gamma_{\mathrm{d}}^{+}}\right)}{\gamma^{-1}\left(\alpha^{-1} K_{\Gamma_{\mathrm{d}}^{-}}+(1-\alpha)^{-1} K_{\Gamma_{\mathrm{d}}^{+}}\right)}
$$

After substituting Eq. 8 in Eq. 4, the discrete flux at $\Gamma_{\mathrm{d}}^{-}$becomes

$$
\begin{aligned}
\mathbf{q}^{-} \cdot \mathbf{n}^{-}= & (1-\omega)\left(\mathbf{q}^{-} \cdot \mathbf{n}^{-}+\gamma^{-1} K_{\Gamma_{\mathrm{d}}}\left(T_{\Gamma_{\mathrm{d}}^{-}}-T_{\Gamma_{\mathrm{d}}^{+}}\right)\right) \\
& +\omega(1-\omega)\left(\alpha^{-1} K_{\Gamma_{\mathrm{d}}^{-}}+(1-\alpha)^{-1} K_{\Gamma_{\mathrm{d}}^{+}}\right)^{-1} \alpha^{-1} K_{\Gamma_{\mathrm{d}}^{-}}\left(\bar{q}_{\Gamma_{\mathrm{d}}^{-}}+\bar{q}_{\Gamma_{\mathrm{d}}^{+}}\right)-\omega \bar{q}_{\Gamma_{\mathrm{d}}^{-}}
\end{aligned}
$$

where $K_{\Gamma_{\mathrm{d}}}=\left(\alpha K_{\Gamma_{\mathrm{d}}^{-}}^{-1}+(1-\alpha) K_{\Gamma_{\mathrm{d}}^{+}}^{-1}\right)^{-1}$ is the combined discrete conductivity of the cohesive zone. If the material at both sides of the cohesive interface is identical, i.e., the case of material failure instead of interface failure, we can take $K_{\Gamma_{\mathrm{d}}^{-}}=K_{\Gamma_{\mathrm{d}}^{+}}$. The combined discrete conductivity $K_{\Gamma_{\mathrm{d}}}$ then equals the material conductivity, independent of the value for $\alpha$.

After eliminating the temperature $T_{\Gamma_{\mathrm{d}}}$ at the interface, the discrete flux at $\Gamma_{\mathrm{d}}^{+}$reads:

$$
\begin{aligned}
\mathbf{q}^{+} \cdot \mathbf{n}^{+}= & (1-\omega)\left(\mathbf{q}^{+} \cdot \mathbf{n}^{+}+\gamma^{-1} K_{\Gamma_{\mathrm{d}}}\left(T_{\Gamma_{\mathrm{d}}^{+}}-T_{\Gamma_{\mathrm{d}}^{-}}\right)\right) \\
& +\omega(1-\omega)(1-\alpha)^{-1} K_{\Gamma_{\mathrm{d}}^{+}}\left(\alpha^{-1} K_{\Gamma_{\mathrm{d}}^{-}}+(1-\alpha)^{-1} K_{\Gamma_{\mathrm{d}}^{+}}\right)^{-1}\left(\bar{q}_{\Gamma_{\mathrm{d}}^{-}}+\bar{q}_{\Gamma_{\mathrm{d}}^{+}}\right)-\omega \bar{q}_{\Gamma_{\mathrm{d}}^{+}}
\end{aligned}
$$

Expressions (9) and (10) are the general expressions for the discrete thermal cohesive zone model of a material interface. From Eqs. 9 and 10, we obtain the identity:

$$
\omega\left(\mathbf{q}^{-} \cdot \mathbf{n}^{-}+\mathbf{q}^{+} \cdot \mathbf{n}^{+}\right)+\omega^{2}\left(\bar{q}_{\Gamma_{\mathrm{d}}^{-}}+\bar{q}_{\Gamma_{\mathrm{d}}^{+}}\right)=0
$$

We define

$$
\bar{q}_{\Gamma_{\mathrm{d}}} \equiv \omega\left(\bar{q}_{\Gamma_{\mathrm{d}}^{-}}+\bar{q}_{\Gamma_{\mathrm{d}}^{+}}\right)
$$

The sum of $\bar{q}_{\Gamma_{\mathrm{d}}^{-}}$and $\bar{q}_{\Gamma_{\mathrm{d}}^{+}}$is the total amount of energy exchanged per unit area and time between the fracture and the surrounding material. The fracture only occupies a fraction $\omega$ of the cohesive zone. Therefore, $\bar{q}_{\Gamma_{\mathrm{d}}}$ represents the average heat flow per unit area of the planar cohesive zone and per unit time. $\bar{q}_{\Gamma_{\mathrm{d}}}$ is positive if energy is transferred from the fracture toward the surrounding material. Equation 11 has to hold for every value of $\omega$, therefore:

$$
\mathbf{q}^{-} \cdot \mathbf{n}^{-}+\mathbf{q}^{+} \cdot \mathbf{n}^{+}+\bar{q}_{\Gamma_{\mathrm{d}}}=0
$$

which simply represents the global energy equilibrium over the cohesive region. Timedependent terms are absent since the cohesive region is assumed planar, and consequently 
does not possess a volume nor a thermal capacity. This does not imply that the model can only describe heat flow along the fracture under steady-state conditions. The fracture has a width and consequently also a volume. The energy conservation law, written for the domain of the fracture, can therefore contain time-dependent terms. The interaction between the fracture domain and the surrounding porous medium takes place via the exchange terms $\bar{q}_{\Gamma_{\mathrm{d}}^{-}}$and $\bar{q}_{\Gamma_{\mathrm{d}}^{+}}$. These terms are the link to the discrete models used in the fracture, e.g., the model described in Sect. 2.4.

\subsubsection{Isolated Micro-Crack or Cavity}

In an isolated micro-crack or cavity, external sources of energy are absent. Therefore, the flux via the damaged part of $\Gamma_{\mathrm{d}}^{-}$to the cavity must be equal to the flux from the cavity to $\Gamma_{\mathrm{d}}^{+}$, or more precisely $\bar{q}_{\Gamma_{\mathrm{d}}^{-}}=-\bar{q}_{\Gamma_{\mathrm{d}}^{+}}$. In this case, expressions (9) and (10) simplify to:

$$
\mathbf{q}^{-} \cdot \mathbf{n}^{-}=(1-\omega)\left(\mathbf{q}^{-} \cdot \mathbf{n}^{-}+\gamma^{-1} K_{\Gamma_{\mathrm{d}}}\left(T_{\Gamma_{\mathrm{d}}^{-}}-T_{\Gamma_{\mathrm{d}}^{+}}\right)\right)-\omega \bar{q}_{\Gamma_{\mathrm{d}}^{-}}
$$

and

$$
\mathbf{q}^{+} \cdot \mathbf{n}^{+}=(1-\omega)\left(\mathbf{q}^{+} \cdot \mathbf{n}^{+}+\gamma^{-1} K_{\Gamma_{\mathrm{d}}}\left(T_{\Gamma_{\mathrm{d}}^{+}}-T_{\Gamma_{\mathrm{d}}^{-}}\right)\right)+\omega \bar{q}_{\Gamma_{\mathrm{d}}^{-}}
$$

Summing both expressions and rearranging yields:

$$
\omega\left(\mathbf{q}^{-} \cdot \mathbf{n}^{-}+\mathbf{q}^{+} \cdot \mathbf{n}^{+}\right)=0
$$

which has to hold for every value of $\omega$. Since $\mathbf{n}^{-}=-\mathbf{n}^{+} \equiv \mathbf{n}$, we find $\mathbf{q}^{-}=\mathbf{q}^{+} \equiv \mathbf{q}$, and the discrete thermal cohesive zone model in case of a micro-crack or cavity reads:

$$
-\mathbf{q} \cdot \mathbf{n}=\underbrace{(1-\omega)\left(-\mathbf{q} \cdot \mathbf{n}+\gamma^{-1} K_{\Gamma_{\mathrm{d}}} \llbracket T \rrbracket\right)}_{(\mathrm{i})}+\underbrace{\omega \bar{q}_{\Gamma_{\mathrm{d}}^{-}}}_{(\mathrm{ii})}
$$

with $\llbracket T \rrbracket=T_{\Gamma_{\mathrm{d}}^{+}}-T_{\Gamma_{\mathrm{d}}^{-}}$, the temperature jump across the micro-crack or cavity. Expression (17) clearly states that the total flux (left hand side) consists of (i) a flux through the undamaged material bond (right hand side, first term) and (ii) a flux through the micro-crack or cavity (right hand side, second term). The flux through the undamaged material bonds (i) consists of a continuum and a discrete component. The physical meaning of both terms can be understood by rearranging Eq. 17:

$$
-\omega \mathbf{q} \cdot \mathbf{n}=(1-\omega) \gamma^{-1} K_{\Gamma_{\mathrm{d}}} \llbracket T \rrbracket+\omega \bar{q}_{\Gamma_{\mathrm{d}}^{-}}
$$

Equation 18 shows that a temperature jump cannot occur (i.e., «T] has to be zero) in absence of damage (i.e., if $\omega=0)$. When damage is present $(\omega \neq 0)$, the thermal flux that flows toward the damaged part of the interface $(-\omega \mathbf{q} \cdot \mathbf{n})$ is redistributed. A part of this energy is exchanged with the micro-crack or cavity via the fractured part of the interface $\left(\omega \bar{q}_{\Gamma_{\mathrm{d}}^{-}}\right)$. The remaining heat flows through the undamaged material bond. The magnitude of the latter is proportional to the temperature difference across the material bond.

\subsubsection{Adiabatic Fracture}

If it is assumed that no heat is exchanged between the material and the fracture, or $\bar{q}_{\Gamma_{\mathrm{d}}^{-}}=$ $\bar{q}_{\Gamma_{\mathrm{d}}^{+}}=0$, we obtain the academic limit case of an adiabatic fracture. Even in a vacuum, this 
situation does not occur since heat exchange by means of radiation will still take place. Considering an adiabatic fracture therefore only has theoretical value. In this case, expressions (9) and (10) reduce to:

$$
\mathbf{q}^{-} \cdot \mathbf{n}=(1-\omega)\left(\mathbf{q}^{-} \cdot \mathbf{n}+\gamma^{-1} K_{\Gamma_{\mathrm{d}}}\left(T_{\Gamma_{\mathrm{d}}^{-}}-T_{\Gamma_{\mathrm{d}}^{+}}\right)\right)
$$

and

$$
\mathbf{q}^{+} \cdot \mathbf{n}^{+}=(1-\omega)\left(\mathbf{q}^{+} \cdot \mathbf{n}^{+}+\gamma^{-1} K_{\Gamma_{\mathrm{d}}}\left(T_{\Gamma_{\mathrm{d}}^{+}}-T_{\Gamma_{\mathrm{d}}^{-}}\right)\right)
$$

Equation 16 also holds in this case. Therefore, $\mathbf{q}^{-}=\mathbf{q}^{+} \equiv \mathbf{q}$, and the discrete thermal cohesive zone model in case of an adiabatic fracture reads:

$$
-\mathbf{q} \cdot \mathbf{n}=(1-\omega)\left(-\mathbf{q} \cdot \mathbf{n}+\gamma^{-1} K_{\Gamma_{\mathrm{d}}} \llbracket T \rrbracket\right)
$$

After rearranging, we obtain:

$$
-\omega \mathbf{q} \cdot \mathbf{n}=(1-\omega) \gamma^{-1} K_{\Gamma_{\mathrm{d}}} \llbracket T \rrbracket
$$

Comparing with Eq. 18, it is clear that the total thermal flux toward the damaged part of the interface $(-\omega \mathbf{q} \cdot \mathbf{n})$ now flows through the undamaged material bond. Also here, the magnitude of the flow is proportional to the temperature difference across the material bond, i.e., flow by means of effective conduction.

\subsection{Heat Transfer Across Micro-Cracks, Cavities, and Fractures}

\subsubsection{Model Classification}

We make a distinction between the cohesive zone, i.e., the region in which $0<\omega<1$, and the fracture $(\omega=1)$. The cohesive zone is characterized by micro-cracks and cavities of different sizes. Depending on the amount of damage, the cavities and micro-cracks are isolated features, or are partially interconnected. In contrast to a cohesive zone, a fracture is considered a single, fully-connected, macroscopic entity. The distinction between cohesive zone and fracture is important in the modeling of heat transfer. Because of the relatively large size of a fracture, convective heat flow can occur. For the smaller micro-cracks and cavities in the cohesive zone, it can be argued that heat transfer is conduction-dominated. Furthermore, the volume of the micro-cracks and cavities in the cohesive zone is much smaller than the one of the fracture. Therefore, neglecting thermal capacity of the fluid inside the micro-cracks and cavities can be defended, as a first approximation. However, this assumption is debatable for fractures, certainly if they are filled with a fluid like water. Below, a simplified model for heat transfer across the cohesive zone is given. The use of more advanced discrete models within the proposed framework is briefly discussed, but they are not considered in the examples in the remainder of this article.

\subsubsection{A Simple Model for Heat Transfer Across Micro-Cracks or Cavities}

A micro-crack or cavity can be seen as two parallel surfaces, exchanging heat by means of conduction, convection, and radiation. Due to the limited dimensions, it can be argued that convective heat transfer is less important than conductive and radiative transfer. The gas or liquid inside the micro-crack or cavity is often less conductive than the surrounding solid material-compare for instance the effective conductivity of concrete $(k \approx 1.2-$ $2 \mathrm{~W} /(\mathrm{m} \mathrm{K}))$ with the conductivity of liquid water at $273 \mathrm{~K}$ and at atmospheric pressure $(\lambda \approx$ 
$0.561 \mathrm{~W} /(\mathrm{m} \mathrm{K}))$. For many materials, micro-cracks and cavities therefore act as a thermal resistance. Since the thermal resistance increases with distance, conduction across a microcrack or cavity is more likely to occur than along the micro-crack. A similar argument holds for radiative transfer. Therefore, disregarding the heat flow along the micro-crack or cavity is defendable in a first approximation. Furthermore, the volume of the micro-cracks and cavities is small enough to neglect the capacity of the fluid inside. Under these assumptions, the heat flux across a micro-crack or cavity can by approximated by:

$$
\bar{q}_{\Gamma_{\mathrm{d}}^{+}}=k_{\Gamma_{\mathrm{d}}}\left(T_{\Gamma_{\mathrm{d}}^{-}}-T_{\Gamma_{\mathrm{d}}^{+}}\right)
$$

where $k_{\Gamma_{\mathrm{d}}}$ is the effective thermal conductivity, given by:

$$
k_{\Gamma_{\mathrm{d}}}=\frac{\lambda N u}{\llbracket \tilde{\mathbf{u}} \rrbracket \cdot \mathbf{n}}+\frac{C_{b} F_{T}}{\frac{1}{e_{\Gamma_{\mathrm{d}}^{-}}}+\frac{1}{e_{\Gamma_{\mathrm{d}}^{+}}}-1}
$$

The first term on the right hand side of Eq. 24 describes combined conductionconvection, while the second term expresses radiation between two parallel surfaces. In Eq. $24, \lambda$ is the thermal conductivity of the fluid in the micro-crack or cavity, $N u$ is the dimensionless Nusselt number describing the ratio of convective to conductive heat transfer, $\llbracket \tilde{\mathbf{u}} \rrbracket \cdot \mathbf{n}$ is the dimension of the micro-crack or cavity in the direction of the normal to the discontinuity, $C_{b}=5.67 \mathrm{~W} / \mathrm{m}^{2} \mathrm{~K}^{4}$ is the black body constant, $e_{\Gamma_{\mathrm{d}}^{-}}$and $e_{\Gamma_{\mathrm{d}}^{+}}$are the emission factors of both crack surfaces, and $F_{T}$ is the temperature factor, given by:

$$
F_{T} \approx \frac{4}{100}\left(\frac{T_{\mathrm{m}}}{100}\right)^{3}
$$

where $T_{\mathrm{m}}=1 / 2\left(T_{\Gamma_{\mathrm{d}}^{-}}+T_{\Gamma_{\mathrm{d}}^{+}}\right)$. For dry air at $273 \mathrm{~K}$ and at atmospheric pressure $\lambda \approx$ $0.025 \mathrm{~W} /(\mathrm{mK})$, while for water vapor under the same conditions, $\lambda \approx 0.016 \mathrm{~W} /(\mathrm{mK})$. For liquid water $\lambda \approx 0.561 \mathrm{~W} /(\mathrm{m} \mathrm{K})$.

The effective conductivity needs to be constrained in order to account for crack closure:

$$
\frac{1}{k_{\Gamma_{\mathrm{d}}}}=\max \left(R_{\Gamma_{\mathrm{d}}}^{\text {contact }}, \frac{1}{k_{\Gamma_{\mathrm{d}}}}\right)
$$

with $R_{\Gamma_{\mathrm{d}}}^{\text {contact }}$ the residual thermal resistance upon crack closure, i.e., when $\llbracket \tilde{\mathbf{u}} \rrbracket \cdot \mathbf{n}=0$. According to Eqs. 23-26, the thermal resistance of the fracture increases with increasing fracture width for a given Nusselt number. If the crack width decreases afterward, the thermal resistance will reduce down to the residual resistance $R_{\Gamma_{\mathrm{d}}}^{\text {contact }}$.

The residual resistance results from the formation of micro-cracks during the irreversible damage process. As micro-cracks initiate and coalesce, $R_{\Gamma_{\mathrm{d}}}^{\text {contact }}$ increases, up to a value $R_{\Gamma_{\mathrm{d}}}^{\text {contact, } \omega=1}$ for a fully developed crack. A simple expression that captures the evolution of the residual thermal resistance reads:

$$
R_{\Gamma_{\mathrm{d}}}^{\text {contact }}=\min \left(\frac{1}{k_{\Gamma_{\mathrm{d}}}^{\text {history }}}, R_{\Gamma_{\mathrm{d}}}^{\text {contact }, \omega=1}\right)
$$

with $k_{\Gamma_{\mathrm{d}}}^{\text {history }}$ the minimum effective thermal conductivity (i.e., maximum thermal resistance) ever reached. 


\subsubsection{Advanced Modeling Strategies}

Equations 23-27 provide a simplified model for heat transfer across micro-cracks or cavities. A wide range of more advanced discrete models is available in literature. They can be easily incorporated within the proposed theory by means of the exchange terms $\bar{q}_{\Gamma_{\mathrm{d}}^{-}} \equiv \overline{\mathbf{q}}_{\Gamma_{\mathrm{d}}^{-}} \cdot \mathbf{n}$ and $\bar{q}_{\Gamma_{\mathrm{d}}^{+}} \equiv \overline{\mathbf{q}}_{\Gamma_{\mathrm{d}}^{+}} \cdot \mathbf{n}$. Basically two strategies exist. In a monolithic approach, $\overline{\mathbf{q}}_{\Gamma_{\mathrm{d}}^{-}}$and $\overline{\mathbf{q}}_{\Gamma_{\mathrm{d}}^{+}}$ are substituted by the transfer equations of the discrete model. This approach works best if the contrast in conductivity between fracture and material matrix is limited. If not, it is suggested to use a staggered approach, where the matrix flow and the fracture flow are solved independently, and the exchange terms $\overline{\mathbf{q}}_{\Gamma_{\mathrm{d}}^{-}}$and $\overline{\mathbf{q}}_{\Gamma_{\mathrm{d}}^{+}}$are updated iteratively.

\section{Implementation in a Strong Discontinuity Framework}

\subsection{Strong Form}

Conservation of energy, in absence of energy sources, reads:

$$
\rho c \frac{\partial T}{\partial t}+\nabla \cdot \mathbf{q}=0 \text { in } \Omega
$$

where $\rho$ is the mass density and $c$ is the thermal capacity of the material, and $t$ denotes time. The proposed framework can be used in combination with basically any constitutive equation. For simplicity we assume that effective conduction is adequately described by Fourier's law:

$$
\mathbf{q}=-\mathbf{K}_{\Omega} \nabla T
$$

where $\mathbf{K}_{\Omega}$ denotes the effective thermal conductivity of the intact continuum material.

The essential boundary conditions are imposed on the external boundary $\Gamma_{1}$ (Fig. 4):

$$
T=\bar{T} \quad \text { on } \Gamma_{1}
$$

The natural boundary conditions are imposed on the external boundary $\Gamma_{2}$ with outward normal $\overline{\mathbf{n}}$ (Fig. 4), and are given by:

$$
\mathbf{q} \cdot \overline{\mathbf{n}}=-\bar{q}_{\Gamma_{2}} \text { on } \Gamma_{2}
$$

Along the internal boundaries $\Gamma_{d}^{+}$and $\Gamma_{d}^{-}$(Fig. 4), the proposed cohesive zone model given by the Eqs. 9 and 10 is applied. The model allows imposing natural boundary conditions along $\Gamma_{\mathrm{d}}^{+}$and $\Gamma_{\mathrm{d}}^{-}$. Here, we employ the discrete model equations described in Sect. 2.4.

Fig. 4 Schematic representation of a body $\Omega$ crossed by a displacement discontinuity, with indication of the employed notation

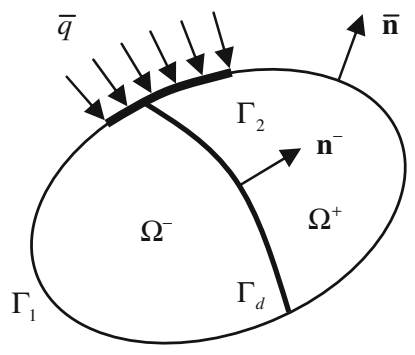




\subsection{Weak Form}

The weak form of Eq. 28 is given by:

$$
\int_{\Omega} w\left(\rho c \frac{\partial T}{\partial t}+\nabla \cdot \mathbf{q}\right) d \Omega=0
$$

which must hold for all admissible variations $w=\hat{w}+H_{\Gamma_{\mathrm{d}}} \tilde{w}$ of temperature $T$. Hereby, $\hat{w}$ and $\tilde{w}$ are smooth, continuous functions on the domain $\Omega$. Following a Galerkin approach leads, after standard manipulations, to the following set of variational statements:

$$
\begin{aligned}
& \int_{\Omega} \hat{w}\left(\rho c \frac{\partial T}{\partial t}\right) \mathrm{d} \Omega+\int_{\Omega} \nabla \hat{w} \cdot \mathbf{K}_{\Omega} \nabla T \mathrm{~d} \Omega \\
& \quad=\int_{\Gamma_{2}} \hat{w} \bar{q}_{\Gamma_{2}} \mathrm{~d} \Gamma-\int_{\Gamma_{\mathrm{d}}^{-}} \hat{w} \mathbf{q}^{-} \cdot \mathbf{n}^{-} \mathrm{d} \Gamma-\int_{\Gamma_{\mathrm{d}}^{+}} \hat{w} \mathbf{q}^{+} \cdot \mathbf{n}^{+} \mathrm{d} \Gamma \\
& \int_{\Omega} H_{\Gamma_{\mathrm{d}}} \tilde{w}\left(\rho c \frac{\partial T}{\partial t}\right) \mathrm{d} \Omega+\int_{\Omega} H_{\Gamma_{\mathrm{d}}} \nabla \tilde{w} \cdot \mathbf{K}_{\Omega} \nabla T \mathrm{~d} \Omega \\
& \quad=\int_{\Gamma_{2}} H_{\Gamma_{\mathrm{d}}} \tilde{w} \bar{q}_{\Gamma_{2}} \mathrm{~d} \Gamma-\int_{\Gamma_{\mathrm{d}}^{-}} H_{\Gamma_{\mathrm{d}}} \tilde{w} \mathbf{q}^{-} \cdot \mathbf{n}^{-} \mathrm{d} \Gamma-\int_{\Gamma_{\mathrm{d}}^{+}} H_{\Gamma_{\mathrm{d}}} \tilde{w} \mathbf{q}^{+} \cdot \mathbf{n}^{+} \mathrm{d} \Gamma
\end{aligned}
$$

where we employed Fourier's law (29) and the boundary conditions (31).

\subsection{Discretized and Linearized Form}

\subsubsection{Discretization in Time}

We employ a finite difference scheme to perform discretization in time:

$$
\frac{\partial T}{\partial t} \approx \frac{T-T^{t}}{\Delta t}
$$

where the superscript $t$ denotes a value at the previous converged time step and $\Delta t$ is the adopted time step.

\subsubsection{Discretization in Space}

Discretization in space is performed using finite elements. Nodes whose support is crossed by a discontinuity are assigned a regular and an enhanced set of degrees of freedom, denoted by $\mathbf{a}$ and $\mathbf{b}$, respectively. The discretized format of the temperature field $T$, given by equation (1), reads:

$$
T=\mathbf{N} \cdot \mathbf{a}+H_{\Gamma_{\mathrm{d}}} \mathbf{N} \cdot \mathbf{b}
$$

where $\mathbf{N}$ is the vector containing the finite element shape functions. The same interpolation is assumed for both regular and enhanced degrees of freedom. Following a Galerkin approach, an identical discretization is performed for the variational field $w$. The gradient of the temperature field and the corresponding variational field can be discretized in a similar fashion 
using the interpolation matrix $\mathbf{B}$, containing the spatial derivatives of the finite element shape functions:

$$
\nabla T=\mathbf{B a}+H_{\Gamma_{\mathrm{d}}} \mathbf{B b}
$$

For elements with only regular degrees of freedom a, the problem field and its gradient are discretized in the standard way.

\subsubsection{Discretized and Linearized Governing Equations}

After applying temporal discretization, according to Eq. 34, and spatial discretization of the variational fields, in analogy with Eqs. 35 and 36, the variational statements (33) read:

$$
\begin{aligned}
& \frac{1}{\Delta t} \int_{\Omega} \mathbf{N}^{T} \rho c T \mathrm{~d} \Omega+\int_{\Omega} \mathbf{B}^{T} \mathbf{K}_{\Omega} \nabla T \mathrm{~d} \Omega=\frac{1}{\Delta t} \int_{\Omega} \mathbf{N}^{T} \rho c T^{t} \mathrm{~d} \Omega \\
& \quad+\int_{\Gamma_{2}} \mathbf{N}^{T} \bar{q}_{\Gamma_{2}} \mathrm{~d} \Gamma-\int_{\Gamma_{\mathrm{d}}^{-}} \mathbf{N}^{T} \mathbf{q}^{-} \cdot \mathbf{n}^{-} \mathrm{d} \Gamma-\int_{\Gamma_{\mathrm{d}}^{+}} \mathbf{N}^{T} \mathbf{q}^{+} \cdot \mathbf{n}^{+} \mathrm{d} \Gamma \\
& \frac{1}{\Delta t} \int_{\Omega} H_{\Gamma_{\mathrm{d}}} \mathbf{N}^{T} \rho c T \mathrm{~d} \Omega+\int_{\Omega} H_{\Gamma_{\mathrm{d}}} \mathbf{B}^{T} \mathbf{K}_{\Omega} \nabla T \mathrm{~d} \Omega=\frac{1}{\Delta t} \int_{\Omega} H_{\Gamma_{\mathrm{d}}} \mathbf{N}^{T} \rho c T^{t} \mathrm{~d} \Omega \\
& \quad+\int_{\Gamma_{2}} H_{\Gamma_{\mathrm{d}}} \mathbf{N}^{T} \bar{q}_{\Gamma_{2}} \mathrm{~d} \Gamma-\int_{\Gamma_{\mathrm{d}}^{-}} H_{\Gamma_{\mathrm{d}}} \mathbf{N}^{T} \mathbf{q}^{-} \cdot \mathbf{n}^{-} \mathrm{d} \Gamma-\int_{\Gamma_{\mathrm{d}}^{+}} H_{\Gamma_{\mathrm{d}}} \mathbf{N}^{T} \mathbf{q}^{+} \cdot \mathbf{n}^{+} \mathrm{d} \Gamma
\end{aligned}
$$

In Eq. 37, the term $\mathbf{q}^{+} \cdot \mathbf{n}^{+}$is given by Eq. 10 and relies on the knowledge of the true local flux vector $\left.\mathbf{q}\right|_{\Gamma_{d}^{+}}$. Even in a medium with constant thermal properties, the magnitude and direction of the heat flux vector can vary from point to point, e.g., when a heat wave travels through the material. Within a finite element context, the spatial variation of the temperature field is described by the derivative of the finite element shape functions (Eq. 36). Except for very specific cases, shape functions do not provide an exact representation of the true spatial variation of the temperature field. Therefore, the local heat flux vector at a point is only approximately known in the finite element method. The true local flux vector $\left.\mathbf{q}\right|_{\Gamma_{\mathrm{d}}^{+}}$can generally not be computed. This conflict can be resolved without approximation if $\omega_{\text {crit }} \leq \omega \leq 1$, where $\omega_{\text {crit }}$ is a real positive number, close to zero. In that case, the flux vector can be eliminated from the cohesive zone model. The reduced format of the discrete thermal model reads:

$\mathbf{q}^{+} \cdot \mathbf{n}^{+}=\left(\frac{1-\omega}{\omega}\right)\left(\gamma^{-1} K_{\Gamma_{\mathrm{d}}}\left(T_{\Gamma_{\mathrm{d}}^{+}}-T_{\Gamma_{\mathrm{d}}^{-}}\right)+\frac{(1-\alpha)^{-1} K_{\Gamma_{\mathrm{d}}^{+}}}{(1-\alpha)^{-1} K_{\Gamma_{\mathrm{d}}^{+}}+\alpha^{-1} K_{\Gamma_{\mathrm{d}}^{-}}} \bar{q}_{\Gamma_{\mathrm{d}}}\right)-\bar{q}_{\Gamma_{\mathrm{d}}^{+}}$

For small values of the damage variable $\left(0 \leq \omega<\omega_{\text {crit }}\right)$, the denominator of the right hand side of Eq. 38 would tend toward zero, leading to large errors in the solution field. Therefore, it is more accurate to employ the original Eq. 10, using an approximate value for the heat flux:

$$
\mathbf{q}^{+}=1 / 2\left(\left.\mathbf{q}\right|_{\Gamma_{\mathrm{d}}^{-}}+\left.\mathbf{q}\right|_{\Gamma_{\mathrm{d}}^{+}}\right)
$$

Similar expressions hold for the term $\mathbf{q}^{-} \cdot \mathbf{n}^{-}$. 
The system of Eq. 37 can be linearized in the classical way. Afterward, the temperature field and its gradient can be substituted by their discretized counterparts (35) and (36). The resulting coupled system of equations after assembly becomes:

$$
\left[\begin{array}{ll}
\mathbf{K}_{a a}^{i} & \mathbf{K}_{a b}^{i} \\
\mathbf{K}_{b a}^{i} & \mathbf{K}_{b b}^{i}
\end{array}\right]\left[\begin{array}{l}
\delta \mathbf{a}^{i+1} \\
\delta \mathbf{b}^{i+1}
\end{array}\right]=\left[\begin{array}{l}
\mathbf{f}_{\text {ext }, a}^{i} \\
\mathbf{f}_{\text {ext }, b}^{i}
\end{array}\right]-\left[\begin{array}{l}
\mathbf{f}_{\text {int }, a}^{i} \\
\mathbf{f}_{\text {int }, b}^{i}
\end{array}\right]
$$

where the superscript $i$ indicates the iteration counter in the global iterative procedure.

The terms of the K-matrix are given by:

$$
\begin{aligned}
\mathbf{K}_{a a}^{i}= & \frac{1}{\Delta t} \int_{\Omega} \mathbf{N}^{T} \rho c \mathbf{N d} \Omega+\int_{\Omega} \mathbf{B}^{T} \mathbf{K}_{\Omega} \mathbf{B} \mathrm{d} \Omega-\int_{\Gamma_{2}} \mathbf{N}^{T} \frac{\partial \bar{q}_{\Gamma_{2}}}{\partial T} \mathrm{~d} \Gamma-\int_{\Gamma_{\mathrm{d}}} \mathbf{N}^{T} \frac{\partial \bar{q}_{\Gamma_{\mathrm{d}}}}{\partial T} \mathrm{~d} \Gamma \\
\mathbf{K}_{a b}^{i}= & \frac{1}{\Delta t} \int_{\Omega} H_{\Gamma_{\mathrm{d}}} \mathbf{N}^{T} \rho c \mathbf{N d} \Omega+\int_{\Omega} H_{\Gamma_{\mathrm{d}}} \mathbf{B}^{T} \mathbf{K}_{\Omega} \mathbf{B} \mathrm{d} \Omega \\
& -\int_{\Gamma_{2}} H_{\Gamma_{\mathrm{d}}} \mathbf{N}^{T} \frac{\partial \bar{q}_{\Gamma_{2}}}{\partial T} \mathrm{~d} \Gamma-\int_{\Gamma_{\mathrm{d}}} H_{\Gamma_{\mathrm{d}}} \mathbf{N}^{T} \frac{\partial \bar{q}_{\Gamma_{\mathrm{d}}}}{\partial T} \mathrm{~d} \Gamma \\
\mathbf{K}_{b a}^{i}= & \frac{1}{\Delta t} \int_{\Omega} H_{\Gamma_{\mathrm{d}}} \mathbf{N}^{T} \rho c \mathbf{N d} \Omega+\int_{\Omega} H_{\Gamma_{\mathrm{d}}} \mathbf{B}^{T} \mathbf{K}_{\Omega} \mathbf{B} \mathrm{d} \Omega \\
& -\int_{\Gamma_{2}} \mathbf{N}^{T} \frac{\partial \bar{q}_{\Gamma_{2}}}{\partial T} \mathrm{~d} \Gamma+\int_{\Gamma_{\mathrm{d}}} H_{\Gamma_{\mathrm{d}}} \mathbf{N}^{T} \frac{\partial \mathbf{q}^{+} \cdot \mathbf{n}^{+}}{\partial T} \mathrm{~d} \Gamma \\
\mathbf{K}_{b b}^{i}= & \frac{1}{\Delta t} \int_{\Omega} H_{\Gamma_{\mathrm{d}}} H_{\Gamma_{\mathrm{d}}} \mathbf{N}^{T} \rho c \mathbf{N d} \Omega+\int_{\Omega} H_{\Gamma_{\mathrm{d}}} H_{\Gamma_{\mathrm{d}}} \mathbf{B}^{T} \mathbf{K}_{\Omega} \mathbf{B} \mathrm{d} \Omega \\
& -\int_{\Gamma_{2}} H_{\Gamma_{\mathrm{d}}} H_{\Gamma_{\mathrm{d}}} \mathbf{N}^{T} \frac{\partial \bar{q}_{\Gamma_{2}} \mathrm{~d} \Gamma+\int_{\Gamma_{\mathrm{d}}} H_{\Gamma_{\mathrm{d}}} H_{\Gamma_{\mathrm{d}}} \mathbf{N}^{T} \frac{\partial \mathbf{q}^{+} \cdot \mathbf{n}^{+}}{\partial T} \mathrm{~d} \Gamma}{}
\end{aligned}
$$

where we introduced $\bar{q}_{\Gamma_{\mathrm{d}}}$ based on Eq. 13 and we used $H_{\Gamma_{\mathrm{d}}^{-}}=0$. The entries in the external force vector read:

$$
\begin{aligned}
\mathbf{f}_{\mathrm{ext}, a}^{i} & =\int_{\Gamma_{2}} \mathbf{N}^{T} \bar{q}_{\Gamma_{2}}^{i} \mathrm{~d} \Gamma \\
\mathbf{f}_{\mathrm{ext}, b}^{i} & =\int_{\Gamma_{2}} H_{\Gamma_{\mathrm{d}}} \mathbf{N}^{T} \bar{q}_{\Gamma_{2}}^{i} \mathrm{~d} \Gamma
\end{aligned}
$$

The entries of the internal force vector are given by:

$$
\begin{aligned}
\mathbf{f}_{\text {int }, a}^{i}= & \frac{1}{\Delta t} \int_{\Omega} \mathbf{N}^{T} \rho\left(c^{i} T^{i}-c^{t} T^{t}\right) \mathrm{d} \Omega-\int_{\Omega} \mathbf{B}^{T} \mathbf{q}^{i} \mathrm{~d} \Omega-\int_{\Gamma_{\mathrm{d}}} \mathbf{N}^{T} \bar{q}_{\Gamma_{\mathrm{d}}}^{i} \mathrm{~d} \Gamma \\
\mathbf{f}_{\text {int }, b}^{i}= & \frac{1}{\Delta t} \int_{\Omega} H_{\Gamma_{\mathrm{d}}} \mathbf{N}^{T} \rho\left(c^{i} T^{i}-c^{t} T^{t}\right) \mathrm{d} \Omega-\int_{\Omega} H_{\Gamma_{\mathrm{d}}} \mathbf{B}^{T} \mathbf{q}^{i} \mathrm{~d} \Omega \\
& +\int_{\Gamma_{\mathrm{d}}^{+}} H_{\Gamma_{\mathrm{d}}} \mathbf{N}^{T}\left(\mathbf{q}^{+} \cdot \mathbf{n}^{+}\right)^{i} \mathrm{~d} \Gamma
\end{aligned}
$$


In the system of Eq. 40, the terms related to the linearization of the non-linear material properties have been disregarded in order to obtain a symmetric system of equations. Hereby, the computational cost per iteration is reduced, but the convergence rate decreases as well. According to Janssen et al. (2007), the former effect dominates for 2D and 3D computations.

\section{Academic Examples}

In this section, two numerical examples are presented. In the first example, the transient thermal behavior of a fractured porous medium is studied. The influence of the damage parameter and the heat capacity are investigated. Next, a similar test is performed on a two-layer composite material. It is shown that impact of damage on the dynamic thermal response depends on the layer order.

\subsection{Heat Transfer in a Fractured Porous Medium}

Transient heat transfer is studied on a 2D square sample with edge length of $0.03 \mathrm{~m}$ (Fig. 5). The sample is discretized with nine square bilinear elements (Q4). A strong discontinuity with a normal pointing in the positive $x$-direction is inserted in the three middle elements. Initially the temperature of the sample is uniform and equals $283 \mathrm{~K}$. The temperature of the nodes on the left boundary is kept constant in time $(\bar{T}=283 \mathrm{~K})$. At $t=0$, the right boundary is exposed to an indoor environment at $T_{i}=293 \mathrm{~K}$. The resulting heat flux is proportional to the difference in temperature between the sample boundary and the environment, and is consequently varying over time:

$$
\bar{q}_{\Gamma_{2}}=h_{i}\left(T_{i}-T\right)
$$

A heat transfer coefficient of $h_{i}=8 \mathrm{~W} /\left(\mathrm{m}^{2} \mathrm{~K}\right)$ is selected. We investigate the influence of the heat capacity $(c=0$ or $500 \mathrm{~J} /(\mathrm{kg} \mathrm{K}))$ and the amount of interface damage $(\omega=0$, 0.1 or 1 ) on the thermal behavior. The case $c=0$ corresponds to the steady-state solution. A mass density of $\rho=2000 \mathrm{~kg} / \mathrm{m}^{3}$ and effective thermal conductivity of $k=0.4 \mathrm{~W} / \mathrm{m} \mathrm{K}$ were used. In this example, we want to focus on the influence of damage on the magnitude of the temperature jump across the discontinuity under steady-state and transient conditions. To simplify interpretation of the results, the fracture is assumed adiabatic $\left(\bar{q}_{\Gamma_{\mathrm{d}}^{-}}=\bar{q}_{\Gamma_{\mathrm{d}}^{+}}=0\right)$. All energy is therefore transported via the undamaged part of the material interface (see Sect. 2.3.3). In the subsequent example, more realistic thermal behavior of the fracture will be considered.

Figure 6 summarizes the results of the simulations. In the left column, the temperature evolution over time is shown for four different locations, namely at the left sample boundary $(x=0 \mathrm{~m})$, at both sides of the discontinuity $(x=0.015 \mathrm{~m})$ and at the right sample boundary

Fig. 5 Heat transfer in a fractured porous medium: geometry, mesh, and boundary conditions

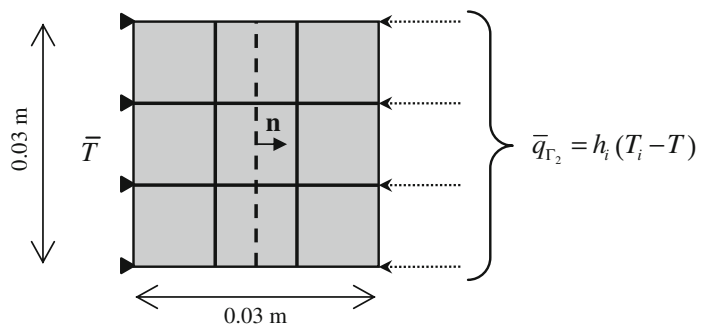



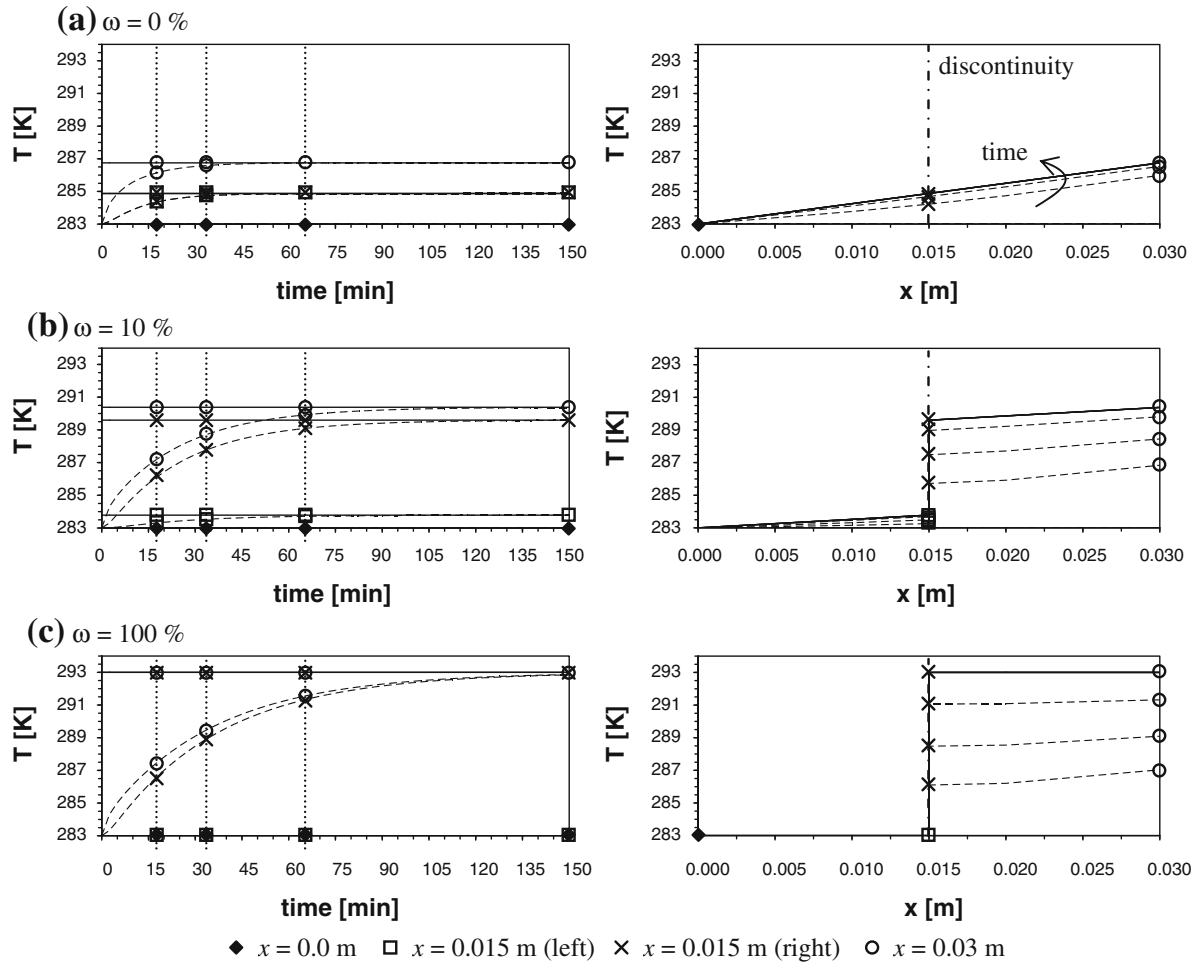

Fig. 6 Heat transfer in a fractured porous medium: temperature profiles at selected points in space (left column) and temperature evolution at selected points in time (right column) for three different amounts of damage at the interface: a $\omega=0$, b 10 , and c $100 \%$. Solid lines indicate steady-state simulations $(c=0 \mathrm{~J} /(\mathrm{kg} \mathrm{K}))$; dashed lines correspond to transient simulations $(c=500 \mathrm{~J} /(\mathrm{kg} \mathrm{K}))$

$(x=0.03 \mathrm{~m})$. In the right column, temperature profiles over the width of the sample are given at selected points in time $(t=0,15,30$ and $60 \mathrm{~s})$.

Figure $6 \mathrm{a}$ shows the results for a damage-free discontinuity. At every instance in time, the temperature profile is continuous across the sample. This indicates that an undamaged interface does not possess thermal resistance. Under steady-state conditions, the temperature at the right sample boundary can be computed analytically from the energy equilibrium:

$$
h_{i}\left(T_{i}-T\right)+\frac{k}{d}(\bar{T}-T)=0 \rightarrow T=\left(h_{i}+\frac{k}{d}\right)^{-1}\left(h_{i} T_{i}+\frac{k}{d} \bar{T}\right)
$$

with $d$ the total thickness of the material sample. For the given material properties and geometry, a temperature of $286.75 \mathrm{~K}$ is obtained. The same value is found in the steady-state simulation. The transient simulation tends asymptotically toward the equilibrium state.

Upon increasing the amount of interface damage, the part of the cross-sectional area that participates in the heat transfer across the discontinuity decreases and consequently the thermal resistance of the interface increases. Correspondingly, the temperature difference over the sample increases and a temperature jump develops across the interface (Fig. 6b). Again, we notice that the transient simulation tends asymptotically toward the steady-state temperature distribution. 


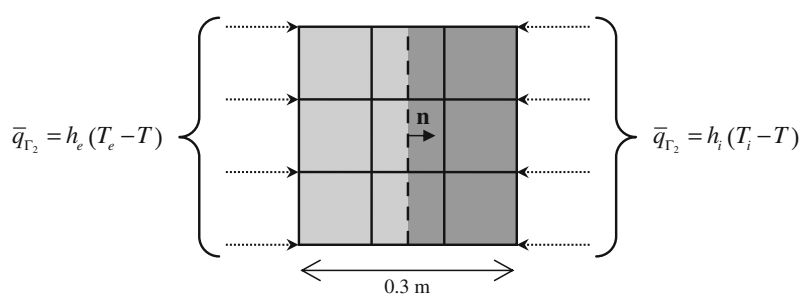

Fig. 7 Heat transfer in a two-layer composite material: geometry, mesh, and boundary conditions

Figure $6 \mathrm{c}$ illustrates the fully fractured case. Since the fracture is considered adiabatic in this simulation, a fully damaged crack acts as a perfect heat barrier. Therefore, the left part of the sample remains at the initial temperature, while the right part of the sample strives toward thermal equilibrium with the environment. The temperature jump across the discontinuity eventually becomes equal to the temperature difference between $T_{i}$ and $\bar{T}$.

This academic example illustrates that the predicted change of the temperature distribution across and the heat flux within the porous medium during the development of a discrete crack exhibits the expected behavior. Note that a time and fracture width-dependent behavior was observed, although the fracture does not have a thermal resistance nor a heat capacityadiabatic behavior was assumed.

\subsection{Heat Transfer in a Composite}

Heat transfer in a two-layer composite material is studied to illustrate that the proposed discrete model is capable of capturing dynamic effects. For a given amount of damage at the interface, it is shown that its influence on the dynamic thermal behavior is not fixed, but depends on the properties of both layers and the layer order.

We consider a 2D square sample with edge length of $0.3 \mathrm{~m}$ (Fig. 7). The sample is discretized with nine square bilinear elements (Q4). A strong discontinuity with normal pointing in the positive $x$-direction is inserted in the three middle elements, separating material 1 (left) from material 2 (right). Both materials have a mass density of $\rho=2000 \mathrm{~kg} / \mathrm{m}^{3}$. The effective thermal conductivity $k_{i}$ and the heat capacity $c_{i}$ of each layer vary from simulation to simulation (Table 1). We will investigate both perfect and imperfect contact between both materials. The imperfect contact is simulated by assuming $10 \%$ interface damage. The thermal behavior of the air-filled cavities between both materials is modeled according to Eqs. 23-25. We assume $e_{\Gamma_{\mathrm{d}}^{-}}=e_{\Gamma_{\mathrm{d}}^{+}}=0.9, N u=1$ and $\llbracket \tilde{\mathbf{u}} \rrbracket \cdot \mathbf{n}=2 \cdot 10^{-3} \mathrm{~m}$. Crack closure is not considered, hence Eqs. 26-27 are not used. All investigated cases are summarized in Table 1. Initially the temperature of the composite sample is uniform and equals $283 \mathrm{~K}$. The left boundary is exposed to an exterior environment with a periodically varying temperature $T_{\mathrm{e}}=283+10 \sin (2 \pi t / 86400)$, corresponding to a simplified day-night cycle $\left(h_{\mathrm{e}}=\right.$ $\left.23 \mathrm{~W} /\left(\mathrm{m}^{2} \mathrm{~K}\right)\right)$.

$$
\bar{q}_{\Gamma_{2}}=h_{\mathrm{e}}\left(T_{\mathrm{e}}-T\right)
$$

The right boundary is in contact with an indoor environment at a constant temperature $T_{\mathrm{i}}=293 \mathrm{~K}\left(h_{\mathrm{i}}=8 \mathrm{~W} /\left(\mathrm{m}^{2} \mathrm{~K}\right)\right.$, see Eq. 44$)$.

We simulate ten cycles, sufficient to reach periodic response of the two-layer composite system. The last cycle is used to compute the amplitude $A_{\mathrm{i}}$ and the phase angle $\phi_{\mathrm{i}}$ of the harmonic temperature fluctuation at the inner surface. The last two columns of Table 1 
Table 1 Investigated parameter combinations and resulting amplitude and phase shift

\begin{tabular}{|c|c|c|c|c|c|c|c|}
\hline & $c_{1}(\mathrm{~J} /(\mathrm{kg} \mathrm{K}))$ & $k_{1}(\mathrm{~W} /(\mathrm{m} \mathrm{K}))$ & $\omega$ & $c_{2}(\mathrm{~J} /(\mathrm{kg} \mathrm{K}))$ & $k_{2}(\mathrm{~W} /(\mathrm{m} \mathrm{K}))$ & $A_{\mathrm{i}}$ & $\phi_{\mathrm{i}}\left(^{\circ}\right)$ \\
\hline \multicolumn{8}{|c|}{ (a) } \\
\hline 1 & 1000 & 1 & 0.0 & 100 & 1 & 2.65 & -81.4 \\
\hline 2 & 1000 & 1 & 0.1 & 100 & 1 & 2.57 & -91.8 \\
\hline 3 & 100 & 1 & 0.0 & 1000 & 1 & 2.22 & -93.2 \\
\hline 4 & 100 & 1 & 0.1 & 1000 & 1 & 2.07 & -102.4 \\
\hline \multicolumn{8}{|c|}{ (b) } \\
\hline 1 & 1000 & 1 & 0.0 & 1000 & 2 & 1.81 & -118.3 \\
\hline 2 & 1000 & 1 & 0.1 & 1000 & 2 & 1.68 & -138.0 \\
\hline 3 & 1000 & 2 & 0.0 & 1000 & 1 & 2.02 & -119.0 \\
\hline 4 & 1000 & 2 & 0.1 & 1000 & 1 & 1.86 & -137.3 \\
\hline
\end{tabular}

Tests are subdivided in two groups (a, b). Corresponding results are shown in Fig. 8a,b

(a) variable capacity

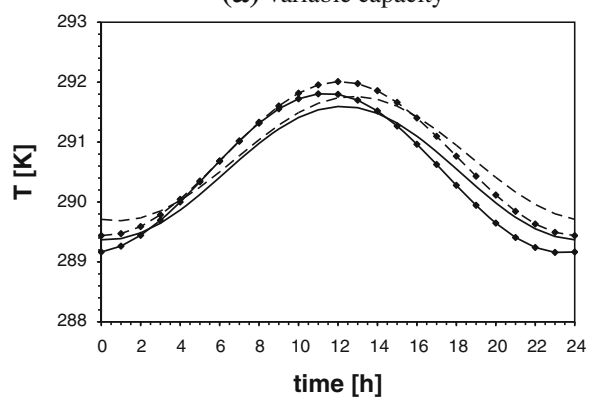

(b) variable conductivity

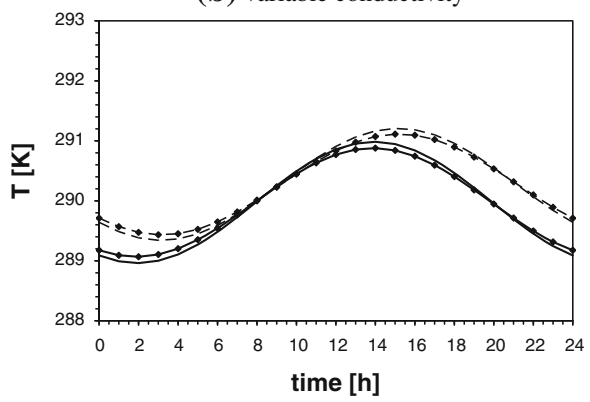

Fig. 8 Heat transfer in a two-layer composite material: temperature fluctuations at the inner surface. Both layers have different a capacity or $\mathbf{b}$ effective conductivity. Solid and dashed lines correspond to simulations with a damage-free and 10\% damaged interface, respectively. All parameter combinations are listed in Table 1. Markers have been added to distinguish between cases 1 and 2 (with markers) and cases 3 and 4 (without markers)

summarize the results. The temperature variation at the inner surface for the last cycle is shown in Fig. 8.

First, we focus on the impact of the amount of interface damage. It is clear that increasing the damage leads to smaller temperature fluctuations (Table 1) and a higher average temperature (Fig. 8) on the inner surface. This is evident, since additional damage corresponds to supplementary contact resistance. But, because the additional damage alters the dynamic behavior of the system, an extra phase shift $\phi_{\mathrm{i}}$ is observed as well, although no capacity is added (Table 1).

It is well known that the dynamics of a layered system depends on the layer order. From Fig. 8 and Table 1, we observe that the effect of a constant increase in damage depends on the layer order. Having a capacitive material at the outside suppresses the damage-induced damping and phase shift $\phi_{\mathrm{i}}$ to some extent (group a). The mutual position of a conductive and a resistive layer does not have a pronounced impact on the effect of the damage on the damping. Damage causes a larger phase shift if the more conductive material is placed at the outside (group b). 


\section{Conclusions}

It was shown that the influence of fractures or partially damaged material interfaces on heat transfer can be modeled adequately by means of a continuous-discontinuous strategy. Without introducing artificial parameters, the proposed cohesive zone model insures a gradual transition from conduction-dominated transfer in the continuum state, prior to crack formation, to convection and radiation-dominated transfer in the damaged state afterward. Hereby, both the continuum model and the discrete model can be chosen freely as a function of the application and the material under study. The thermal cohesive zone model can be directly linked to a mechanical cohesive zone model by means of a damage variable. In absence of damage, the continuum behavior is recovered. The methodology proved to be applicable for quasi-static, periodic, and transient problems.

Acknowledgment Funding for this study was provided by the Flemish Institute for Science and Technology (IWT-SBO project 30175).

\section{References}

Alfaiate, J., Moonen, P., Sluys, L.J., Carmeliet, J.: On the use of strong discontinuity formulations for the modeling of preferential moisture uptake in fractured porous media. Comput. Methods Appl. Mech. Eng. (2010). doi:10.1016/j.cma.2010.05.004

Barenblatt, G.I.: The mathematical theory of equilibrium cracks in brittle fracture. Adv. Appl. Mech. 7, 55-129 (1962)

Carmeliet, J., Delerue, J.-F., Vandersteen K., Roels, S.: Three-dimensional liquid transport in concrete cracks. Int. J. Numer. Anal. Methods Geomech. (2004). doi:10.1002/nag.373

De Boer, R.: Theory of Porous Media-Highlights in the Historical Development and Current State. Springer, Berlin (2000)

de Borst, R., Remmers, J.J.C., Needleman, A., Abellan, M.-A.: Discrete vs smeared crack models for concrete fracture: bridging the gap. Int. J. Numer. Anal. Methods Geomech. (2004). doi:10.1002/nag.374

Dugdale, D.S.: Yielding of steel sheets containing slits. J. Mech. Phys. Solids 8, 100-104 (1960)

Fagerström, M., Larsson, R.: A thermo-mechanical cohesive zone formulation for ductile fracture. J. Mech. Phys. Solids (2008). doi:10.1016/j.jmps.2008.06.002

Hillerborg, Modeer, M., Pettersson, P.E.: Analysis of crack formation and crack growth in concrete by means of fracture mechanics and finite elements. Cem. Concr. Res. (1976). doi:10.1016/0008-8846(76)90007-7

Janssen, H., Blocken, B., Carmeliet, J.: Conservative modeling of the moisture and heat transfer in building components under atmospheric excitation. Int. J. Heat Mass Transf. (2007). doi:10.1016/j. ijheatmasstransfer.2006.06.048

Jirásek, M., Zimmermann, T.: Embedded crack model. Part II: combination with smeared cracks. Int. J. Numer. Methods Eng. (2001). doi:10.1002/1097-0207(20010228)50:6<1291::AID-NME12>3.0.CO;2-Q

Melenk, J.M., Babuška, I.: The partition of unity finite element method: basic theory and applications. Comput. Methods Appl. Mech. Eng. (1996). doi:10.1016/S0045-7825(96)01087-0

Moës, N., Dolbow, J., Belytschko, T.: A finite element method for crack growth without remeshing. Int. J. Numer. Methods Eng. (1999). doi:10.1002/(SICI)1097-0207(19990910)46:1<131::AID-NME726>3.0. $\mathrm{CO} ; 2-\mathrm{J}$

Moonen, P., Carmeliet, J., Sluys, L.J.: A continuous-discontinuous approach to simulate fracture processes in quasi-brittle materials. Philos. Mag. (2008). doi:10.1080/14786430802566398

Moonen, P., Sluys, L.J., Carmeliet, J.: A continuous-discontinuous approach to simulate physical degradation processes in porous media. Int. J. Numer. Methods Eng. (2010). doi:10.1002/nme.2924

Pruess, K., Oldenburg, C., Moridis, G.: TOUGH2 User's Guide, Version 2.0, Lawrence Berkeley National Laboratory Report LBNL-43134, Berkeley, November (1999)

Ren, Z., Bićanić, N.: Simulation of progressive fracturing under dynamic loading conditions. Commun. Numer. Methods Eng. (1997). doi:10.1002/(SICI)1099-0887(199702)13:2<127::AID-CNM44>3.0.CO;2-8

Réthoré, J., de Borst, R., Abellan, M.-A.: A two-scale approach for fluid flow in fractured porous media. Int. J. Numer. Methods Eng. (2007). doi:10.1002/nme.1962 
Réthoré, J., de Borst, R., Abellan, M.-A.: A two-scale model for fluid flow in an unsaturated porous medium with cohesive cracks. Comput. Mech. (2008). doi:10.1007/s00466-007-0178-6

Roels, S., Vandersteen, K., Carmeliet, J.: Measuring and simulating moisture uptake in a fractured porous medium. Adv. Water Resour. (2003). doi:10.1016/S0309-1708(02)00185-9

Roels, S., Moonen, P., de Proft, K., Carmeliet, J.: A coupled discrete-continuum approach to simulate moisture effects on damage processes in porous materials. Comput. Methods Appl. Mech. Eng. (2006). doi:10. 1016/j.cma.2005.05.051

Secchi, S., Simoni, L., Schrefler, B.A.: Cohesive fracture growth in a thermoelastic bi-material medium. Comput. Struct. (2004). doi:10.1016/j.compstruc.2004.03.059

Segura, J.M., Carol, I.: Coupled HM analysis using zero-thickness interface elements with double nodes. Part I: theoretical model. Int. J. Numer. Anal. Methods Geomech. (2008). doi:10.1002/nag.735

Simone, Wells, G.N., Sluys, L.J.: From continuous to discontinuous failure in a gradient-enhanced continuum damage model. Comput. Methods Appl. Mech. Eng. (2003). doi:10.1016/S0045-7825(03)00428-6

Therrien, R., Sudicky, E.A.: Three-dimensional analysis of variably-saturated flow and solute transport in discretely-fractured porous media. J. Contam. Hydrol. (1996). doi:10.1016/0169-7722(95)00088-7

Vandersteen, K., Carmeliet, J., Feyen, J.: A network approach to derive unsaturated hydraulic properties of a roughwalled fracture. Transp. Porous Med. (2003). doi:10.1023/A:1021150732466

Wells, G.N., Sluys, L.J.: A new method for modeling cohesive cracks using finite elements. Int. J. Numer. Methods Eng. (2001). doi:10.1002/nme.143

Wu, Y.S., Haukwa, C., Bodvarsson, G.S.: A site-scale model for fluid and heat flow in the unsaturated zone of Yucca Mountain, Nevada. J. Contam. Hydrol. (1999). doi:10.1016/S0169-7722(99)00014-5 